\title{
Daily Inoculum Levels of Gibberella zeae on Wheat Spikes
}

L. Francl, North Dakota State University, Fargo; G. Shaner, Purdue University, West Lafayette, IN; G. Bergstrom, Cornell University, Ithaca, NY; J. Gilbert, Agriculture and Agri-Food Canada, Manitoba; W. Pedersen, University of Illinois, Urbana; R. Dill-Macky, University of Minnesota, St. Paul; L. Sweets and B. Corwin, University of Missouri-Columbia; Y. Jin and D. Gallenberg, South Dakota State University, Brookings, and J. Wiersma, University of Minnesota, Crookston

\begin{abstract}
Francl, L., Shaner, G., Bergstrom, G., Gilbert, J., Pedersen, W., Dill-Macky, R., Sweets, L., Corwin, B., Jin, Y., Gallenberg, D., and Wiersma, J. 1999. Daily inoculum levels of Gibberella zeae on wheat spikes. Plant Dis. 83:662-666.

The inoculum level of Gibberella zeae on wheat spikes was measured during 1995 and 1996 in nine locations of Canada and the United States prone to Fusarium head blight of wheat. Spikes were exposed after exsertion and until kernel milk or soft dough stage in fields with wheat or corn residue as a source of inoculum; other spikes were exposed in a location remote from any obvious inoculum source; and in 1995 only, control plants remained in a greenhouse. After $24 \mathrm{~h}$, spikes were excised and vigorously shaken in water to remove inoculum. Propagules were enumerated on selective medium and identified as G. zeae from subcultures. Significantly more inoculum was detected from fields in epidemic areas than from remote sites in an epidemic and from fields in nonepidemic areas. The median inoculum level was $20 \mathrm{CFU}$ of G. zeae per spike per day in fields experiencing an epidemic, $4 \mathrm{CFU}$ in locations remote from epidemic fields, 2 $\mathrm{CFU}$ in nonepidemic fields, and $1 \mathrm{CFU}$ in locations remote from a source of inoculum in nonepidemic areas. In an epidemic region, inoculum levels near corn stubble reached up to 587 CFU of G. zeae per spike per day, and the median inoculum level of $126 \mathrm{CFU}$ was significantly higher than the median of $13 \mathrm{CFU}$ found near wheat residue. Inoculum was not detected or occurred sporadically during extended dry periods. While inoculum increased during rainy periods, timing of increased levels was variable. Fusarium head blight epidemics were associated with multiple inoculation episodes and coincident wet periods.
\end{abstract}

Additional keywords: Fusarium graminearum, scab

Fusarium head blight or scab of wheat (Triticum aestivum L. em. Thell) is incited primarily by ascospores of Gibberella zeae (Schwein.) Petch and conidia of its anamorph, Fusarium graminearum Schwabe $(2,8,10,15)$. Serious epidemics have occurred during recent growing seasons in the spring wheat, durum (T. turgidum var. durum Desf.), and barley (Hordeum vulgare L.) production area in the northern Great Plains and in the soft winter wheat area in eastern Canada and the United States. Estimated losses of wheat and barley exceeded 16.5 million metric tons between 1991 and 1996 in these areas (7).

G. zeae produces ascospores in perithecia borne on plant residues. This primary inoculum presumably causes most of the economic loss in wheat (2). Sporulation may recur for several years as debris de-

Corresponding author: L. J. Francl

E-mail: francl@badlands.nodak.edu

Accepted for publication 7 April 1999.

Publication no. D-1999-0428-01R

This article is in the public domain and not copyrightable. It may be freely reprinted with customary crediting of the source. The American Phytopathological Society, 1999. composes (6). Mature ascospores are liberated from perithecia during wet periods and humid evening hours (11) and are dispersed by wind to wheat spikes (5). In contrast, conidia formed on stubble or foliar lesions are thought to be primarily splash dispersed $(7,10)$. Severe Fusarium head blight epidemics result when wheat spikes are infected during anthesis or shortly afterward $(1,10)$. The number of spores deposited on receptive wheat spikes and the relative contribution of ascospores and conidia to crop damage is unknown.

The potential contribution of widely dispersed inoculum needs to be examined because of the large area on which hosts of $G$. zeae are grown under conservation tillage practices in North America. Fusarium head blight incidence declined by $90 \%$ within 5 to $22 \mathrm{~m}$ from a small area source of G. zeae inoculum (5). Although microscale (from $1 \mathrm{~cm}$ to $1 \mathrm{~km}$ ) spore dispersal can be decreased considerably by removal, destruction, or burial of infested residues, the role and importance in Fusarium head blight epidemics of local $(100 \mathrm{~m}$ to $50 \mathrm{~km}$ ) and mesoscale (10 to 200 $\mathrm{km})$ spore dispersal is less well understood.

Disease cycle components of Fusarium head blight are difficult to study in the field because of the unpredictable occur- rence of epidemics. For example, nine moderate to severe outbreaks occurred in a humid region of Argentina during a span of 66 years (9). In contrast, the warm, humid Yangtze Valley of China experiences an epidemic every 2 years on average (10). The location and timing of Fusarium head blight epidemics in the endemic zone of North America (7) fall within this range. Thus, scientists from endemic areas in the United States and Canada cooperated to ensure that field studies were conducted in environments conducive to Fusarium head blight development. The objectives of this work were: (i) to compare the number of spores deposited on wheat spikes in epidemic and nonepidemic areas, (ii) to determine if inoculum could be found on spikes in sites remote from a source, and (iii) to determine if numbers of spores at remote sites were less than those near inoculum sources or differed between epidemic and nonepidemic locations. Data trends uncovered during the course of the study led to a cursory analysis of spore deposition on spikes in relation to rainfall and to corn (Zea mays L.) as the previous crop.

\section{MATERIALS AND METHODS}

Wheat spikes were assayed for Fusarium head blight inoculum during the 1995 growing season in Ithaca, New York (NY), West Lafayette, Indiana (IN), ChampaignUrbana, Illinois (IL), Columbia, Missouri (MO), Brookings, South Dakota (SD), Crookston and St. Paul, Minnesota (MN1 and MN2), Fargo, North Dakota (ND), and Winnipeg, Manitoba (MB). In 1996, the study was conducted in the aforementioned sites except for Crookston. A common protocol was followed by all cooperators, but the protocol regarding exposure of spikes differed between years. Eight to 26 samples were collected annually at each site (Table 1). However, five samples from $\mathrm{ND}$ and one sample from NY collected late in 1996 were excluded from the results because of the potential for an experimental artifact.

1995 protocol. The hard red spring wheat cultivar Butte 86 was planted in greenhouses approximately weekly in 20$\mathrm{cm}$-diameter pots. Plants were thinned to three per pot and fertilized as needed. Plants served as assays for head blight inoculum when they reached anthesis or 
shortly thereafter. One pot was exposed for $24 \mathrm{~h}$ in a wheat field having wheat or corn residue on the surface, one pot was taken to an area remote from inoculum, and one pot was kept in greenhouses as a control. The remote sites were $>0.5 \mathrm{~km}$ from any commercial corn or small grain field and included urban yards, roofs of buildings, and campus grounds. These locations were ranked by their estimated distance from commercial wheat, barley, and corn fields (Table 1). Spikes were exposed between head exsertion and soft dough of the local wheat crop, and the severity of head blight in each area was noted. Twice in NY and once in IN, spikes were exposed for three days over a weekend; in these cases, counts were averaged to estimate daily inoculum level. Environmental data were collected from meteorological stations nearest field sites.

Immediately after exposure, spikes from the three main culms were harvested, placed in a container with $30 \mathrm{ml}$ of sterile distilled water, and shaken vigorously for 1 min. Three to five replicate $1-\mathrm{ml}$ aliquots were spread on petri plates containing Komada's selective medium (4) and allowed to dry under a sterile air flow. Plates were incubated in the dark for 2 to 3 days, followed by an alternating cycle of 12-h light and 12-h dark. After approximately 2 weeks incubation at 20 to $22^{\circ} \mathrm{C}$, colonies were typed as to morphology (color and growth habit) and counted. Typed colonies were transferred serially to potato dextrose agar (PDA) and carnation leaf agar (5) in numbers proportional to their frequency on selective medium. Axenic sporulating cultures were speciated on carnation leaf agar. The number of typed colonies was multiplied by the percentage speciated as $G$. zeae to derive the number of $G$. zeae CFU per spike per day. In MN1 and IL, colonies were identified to genus, and in $\mathrm{MB}, \mathrm{ND}$, and NY, investigators speciated colonies other than G. zeae.

1996 protocol. Wheat or corn stubble from 1995 served as a reservoir of inoculum and locally adapted, Fusarium head

Table 1. Locations and number of sample days in 1995 and 1996 that wheat spikes were exposed to Gibberella zeae inoculum in fields and remote sites ${ }^{\mathrm{a}}$

\begin{tabular}{lcc}
\hline & \multicolumn{2}{c}{ No. of samples } \\
\cline { 2 - 3 } Location & $\mathbf{1 9 9 5}$ & $\mathbf{1 9 9 6}$ \\
\hline Crookston, Minnesota & 9 & 0 \\
Fargo, North Dakota & 19 & 13 \\
Urbana, Illinois & 8 & 10 \\
West Lafayette, Indiana & 14 & 16 \\
Brookings, South Dakota & 12 & 16 \\
Columbia, Missouri & 11 & 9 \\
Ithaca, New York & 11 & 20 \\
Winnipeg, Manitoba & 22 & 11 \\
St. Paul, Minnesota & 26 & 9 \\
\hline
\end{tabular}

${ }^{a}$ Locations were ranked by proximity of their remote sites to commercial wheat and corn fields. blight susceptible wheat cultivars (Clark [IL, IN, MO], Harus [NY], or Norm [MB, $\mathrm{MN}$ 2, ND, SD]) were grown in a field larger than 1 ha following normal agronomic practices. Glassine bags, normally used in wheat breeding, were secured over spikes of about 300 culms in the boot stage. Five arbitrarily chosen spikes were unbagged for $24 \mathrm{~h}$ beginning as early as three-fourths spike exsertion and ending as late as milk to soft dough. Simultaneously, peduncles of five bagged culms were cut several centimeters below the spike, and the cut end was placed in water and taken to a remote site where spikes were exposed. At some sites, unexposed spikes were harvested periodically, and these controls tested negative for Fusarium sp. Head blight severity of the wheat crop in the field was recorded in the soft dough stage. This protocol modification was tested at IN for 11 days during 1995, and results were comparable to the 1995 protocol in detection of two major inoculation events; however, the 1995 protocol had a higher count than the new protocol ( 85 versus $4 \mathrm{CFU}$ per spike) during a third event.

Spikes were vigorously shaken for $1 \mathrm{~min}$ in $50 \mathrm{ml}$ of sterile distilled water. A sample of 0.5 or $1.0 \mathrm{ml}$ was transferred to three to five replicate plates of Komada's selective medium. Fungal colonies were classified as to color and growth habit after 2 weeks of growth under alternating light and dark periods at 20 to $22^{\circ} \mathrm{C}$. Type colonies were transferred to PDA in numbers proportional to their frequency on selective medium. Pure isolates were then transferred to carrot agar to promote the homothallic production of perithecia diagnostic for $G$. zeae (5). Counts of G. zeae colonies were adjusted to CFU per spike per day.

Analysis. Inoculum data were categorized into four classes based on location collected: field sample, epidemic area $(\mathrm{n}=$ 106); field sample, nonepidemic area $(\mathrm{n}=$ 105); remote sample, epidemic area $(\mathrm{n}=$ 90); and remote sample, nonepidemic area $(\mathrm{n}=88)$. These summary data classes were evaluated for normality by KolmogorovSmirmov tests. The nonparametric MannWhitney test of median confidence interval was chosen based on these results. Four a priori treatments were compared: field samples from epidemic and nonepidemic areas, field samples and remote samples in epidemic areas, field and remote samples in a nonepidemic area, and remote samples from epidemic and nonepidemic areas. Inoculum data from corn residue and noncorn residue fields in an epidemic area also were compared.

\section{RESULTS}

1995. Fusarium head blight was greatest in IN and IL, with about $25 \%$ disease severity. MB, MO, ND, and MN1 experienced moderate epidemics, each with about $10 \%$ severity. Very little Fusarium head blight developed in the NY, MN2, or SD sites.

G. zeae was found in all locations, and inoculum levels in areas of severe and moderate Fusarium head blight epidemics ranged from zero to hundreds of G. zeae CFU per spike per day (Fig. 1). Lowest counts were found during the first week of exposure, but beyond this trend, inoculum deposition lacked a distinct seasonal pattern. Inoculum counts were high on days with rain or shortly thereafter, but the precise timing was unpredictable, and gaps in bioassay periods prevented a definitive conclusion.

G. zeae was found in sites remote from known sources of inoculum (Fig. 1). Generally, fewer colonies were recovered from spikes exposed at sites remote from a source, but there were exceptions. Unless the remote site was in a large urban area such as Winnipeg, MB (Fig. 1B), the decrease often was not dramatic. In ND, spikes of control plants left in the greenhouse often yielded propagules of G. zeae after the first week of the study (Fig. 1C).

More than 2,000 CFU of Fusarium spp. per spike per day in a field and about 600 CFU of Fusarium spp. per spike per day in a remote site were recovered on average in IL. MN1 averaged about 1,000 CFU of Fusarium spp. per spike per day in a field. Plants left in the greenhouse served as the remote site at $\mathrm{MN} 1$, and colony counts in those samples averaged about $100 \mathrm{CFU}$ of Fusarium spp. per spike per day. In NY, $F$. subglutinans (Wollenw. \& Rein.) Nelson, Tous. \& Mar., F. sporotrichioides Sherb., $F$. moniliforme (Sheld.), and $F$. proliferatum (Matsu.) were identified in addition to $F$. graminearum. F. sporotrichioides and $F$. avenaceum (Fr.) Sacc. occurred with regularity in $\mathrm{ND}$, while $F$. moniliforme and $F$. equiseti (Corda) Sacc. occurred less frequently. In $\mathrm{MB}, F$. avenaceum, $F$. culmorum (W.G. Smith) Sacc., F. sporotrichioides, and $F$. poae (Peck) Wollenw. were also recovered from spikes. Interestingly, more than 1,700 CFU of $F$. poae per spike were found in MB late in the sampling period on day 208.

1996. Moderate to severe head blight epidemics occurred in the northern and western parts of the soft winter wheat region and in isolated pockets of the northern Great Plains (7). The MO, IL, and IN locations were within the Fusarium head blight epidemic area. The ND location experienced a Fusarium head blight severity of $2 \%$, and the weather in MB, MN2, and SD was hot and dry around flowering, which resulted in no to trace amounts of head blight.

From 0 to $587 \mathrm{CFU}$ of $G$. zeae per spike per day were recovered from wheat spikes exposed in fields near a source of inoculum, with daily amounts of more than 50 CFU per spike common in epidemic areas. Daily inoculum levels of $G$. zeae were highest in corn stubble fields located in 
$\mathrm{MO}$ and IL, where more than $300 \mathrm{CFU}$ per spike per day were observed on several days (Fig. 1D). The IN site was adjacent to a field with corn stubble, and although in an epidemic area, inoculum levels were more comparable to field sites with wheat stubble. The nonepidemic areas of MB and ND had lower counts than epidemic areas (Fig. 1B and F). Inoculum counts were also low in the urban area of St. Paul (MN2), and inoculum levels at the SD site were between 0 to $4 \mathrm{CFU}$ per spike per day, with a single sample of 40 CFU per spike.

Less inoculum often was found in locations remote from a source than in comparable field samples, but as in 1995, several assay periods were exceptions. The highest median inoculum levels from remote locations were in IL with $19 \mathrm{CFU}$ and IN with $24 \mathrm{CFU}$ of G. zeae.
F. equiseti was found in $\mathrm{MB}$ in addition to the species in the aforementioned 1995 assay. In ND, F. sporotrichioides was commonly recovered, but other colony types that occurred less frequently were not speciated.

High levels of inoculum resulted from rainy periods that persisted for several days, and overall, inoculum levels seemed more closely associated with weather patterns than with crop growth stage (Fig. 1). Conversely, dry periods were associated with low inoculum levels. The timing of high inoculum deposition relative to rainfall was variable.

Statistical summary. Data distributions, medians and means were calculated for the classification structure (Fig. 2). Daily inoculum levels on spikes were highly variable within each class, and each was nonnormal according to the KolmogorovSmirmov tests $(P \leq 0.01)$. The data distributions were highly skewed because of zero counts mixed with inoculation events. According to the Mann-Whitney test, the median inoculum level was significantly higher $(P<0.0001)$ in fields from epidemic areas (19.3 CFU) than in fields in nonepidemic areas (2.2 CFU) and remote sites in epidemic areas (4.5 CFU). Similarly, the median level from remote sites in an epidemic area was significantly higher $(P<0.0002)$ than in remote samples from nonepidemic areas (1 CFU). The difference between nonepidemic remote and field sample medians was slight, 1.2 CFU, but significantly different $(P<0.02)$. In epidemic areas, field samples were subclassified as fields with corn or wheat residue, and the median inoculum level from fields with corn residue (126 CFU) was higher $(P=0.0015)$ than from fields with wheat residue (13 CFU).
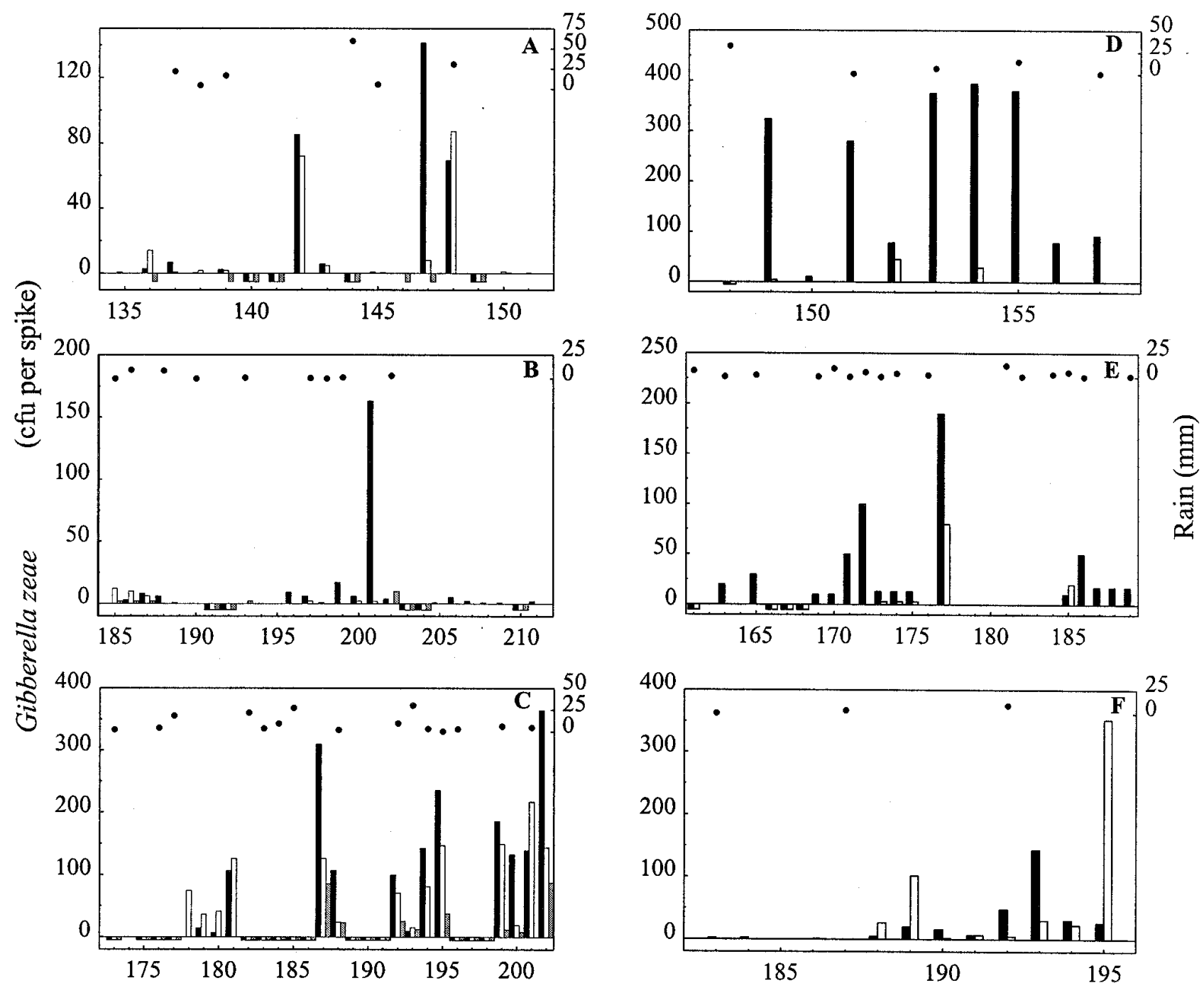

Day of the year

Fig. 1. Daily levels of Gibberella zeae (CFU) on wheat spikes for selected experimental locations that illustrate inoculum under epidemic (>10\% disease severity) and nonepidemic conditions. Spikes were exposed in fields (solid bars), or at sites remote from fields (open bars), or in 1995 only, remained in greenhouses (gray bars). Unsampled periods are shown as negative values to distinguish them from zero inoculum counts. Precipitation is indicated by circles plotted on the right y-axis. The location, years, and disease intensity were: Indiana, 1995 epidemic (A); Manitoba, 1995 epidemic (B); North Dakota, 1995 epidemic (C); Missouri, 1996 epidemic (D); New York, 1996 nonepidemic (E); and North Dakota, 1996 nonepidemic (F). 


\section{DISCUSSION}

These experiments provide the first estimate of inoculum levels of $G$. zeae on wheat spikes under natural conditions. Moderate to severe Fusarium head blight epidemics occurred in several of the experimental locations in each year of the study. Thus, the strategy of experimentation in widely scattered locations was successful in obtaining data from Fusarium head blight epidemic and nonepidemic areas. In 1995, some authors had problems growing potted spring wheat in warm greenhouses, and others had problems speciating fungal colonies. Therefore, the protocol for handling plants and speciation was adjusted in 1996.

Multiple inoculation events consisting of 50 or more CFU of $G$. zeae per spike per day in areas with moderate and severe epidemics suggest that multiple infections contribute to cumulative head blight severity. In contrast, nonepidemic areas were characterized by low average inoculum levels and isolated cases of deposition $>50$ CFU (Fig. 2). Presumably, inoculation events must be followed by wet weather for blight development $(1,10)$. For example, the inoculation on day 201 in MB, 1995, where head blight was moderately severe, was followed by rainfall (Fig. 1B); whereas a comparable amount of inoculum on day 177 was followed by 4 days of dry weather in NY, where no epidemic ensued (Fig. 1E). Although these results are not definitive, they are exemplary of our knowledge of the infection process and suggest a basis for Fusarium head blight forecasting.

The anthers are the most receptive tissue of the spike at anthesis, but other tissue, such as the glumes, also may be penetrated $(3,12,16)$. If the exposed surface area of receptive tissue relative to the total spike is assumed to be about $10 \%$, we can speculate one to several infected spikelets would be probable given an inoculum level of $>50$ propagules, an infection efficiency $>10 \%$, and a suitable environment. Simultaneous infection of several spikelets could accelerate spike colonization through the rachis, leading to more severe damage. Also, if we accept as valid the assumption that 50 propagules make infection probable, the approximate $500 \mathrm{CFU}$ of $G$. zeae per spike found after exposure in a field with corn residue should result in increased incidence and severity compared with other environments. Although the blight intensity might be less than 10 times greater, as might be inferred from the spore level ratio (14), ample evidence exists regarding the inadvisability of growing wheat after corn (17).

Multiple inoculations may have a bearing on strategies for discovery of resistant genotypes. Plant resistance mechanisms are thought to include resistance to spikelet infection and inhibition of growth by the fungus into the rachis (13). Artificial in- oculation by means of infested material spread uniformly throughout an irrigated nursery may simulate epidemic conditions more realistically and may detect growth through the rachis more readily than a single inoculation of spikes (18). Finally, comparable inoculum levels on both awned (Butte 86 and Norm) and awnless wheats (Clark and Harus) argue against any attempt to lessen disease severity through breeding for or against this architectural feature.

Recovery of inoculum at distances remote from any known source of G. zeae propagules indicates that dispersal may occur on the order of kilometers to tens of kilometers or more (Fig. 1). Colonies often could be recovered from control plants in $\mathrm{ND}$ and MN1 greenhouses, which were relatively close to inoculum sources (Table 1), again suggesting widely dispersed spores. The lower frequency of remote inoculation events and approximate $75 \%$ decline in average inoculum counts indicate a dominant role for within-field inoculum sources. Despite this overall picture, 14 inoculation events $>50 \mathrm{CFU}$ from 90 remote samples (Fig. 2B) suggest Fusarium head blight can readily spread from field to field in an epidemic area. Further research is needed to define the (i) assessment of disease severity on plants within an epidemic locale but not exposed to within-field inoculum sources, (ii) measurement of the effect of various resi(iii) defining the relative contribution of ascospores and conidia, and (iv) monitoring of the environmental conditions associrole of widely dispersed spores, including: dues and cropping systems on dispersal,

ated with sporulation and dispersal. If widely dispersed inoculum is a significant factor in epidemic severity, wheat grown in a locale with large areas of infested residue is at risk of infection even though little or no infested residue is present in the field.

Multiple inoculation and mesodistance spore dispersal affect prophylactic disease management options. Essentially, susceptible spikelets must be protected during the period of receptivity to assure a quality product at harvest. Thus, any fungicide or biological agent must have a properly timed application, a highly efficient and persistent activity, and excellent tissue coverage. Furthermore, growers will need to be vigilant for environmental conditions conducive for disease even if they practice recommended rotations and residue management.

High inoculum levels were positively related to rains, but a consistent temporal relationship between inoculum episodes and rainfall was not found in this study. Paulitz (11) found that rain washed spores from the air and ascospore dispersal was greatest 24 to $48 \mathrm{~h}$ after a rain ended. The lack of a temporal relationship between rain and inoculation in the present study may have been due to the indirect measurement of dispersal, imprecise environmental and inoculum measurements, variable abundance of ascospores and conidia, and gaps in the assay periods.

Very high counts of $G$. zeae occurred in ND (example datum shown in Fig. 1F) and NY ( $10^{3}$ CFU per spike per day) among the lattermost samples of the season. Occurrences were not associated with rainfall, location, or epidemic severity, suggesting

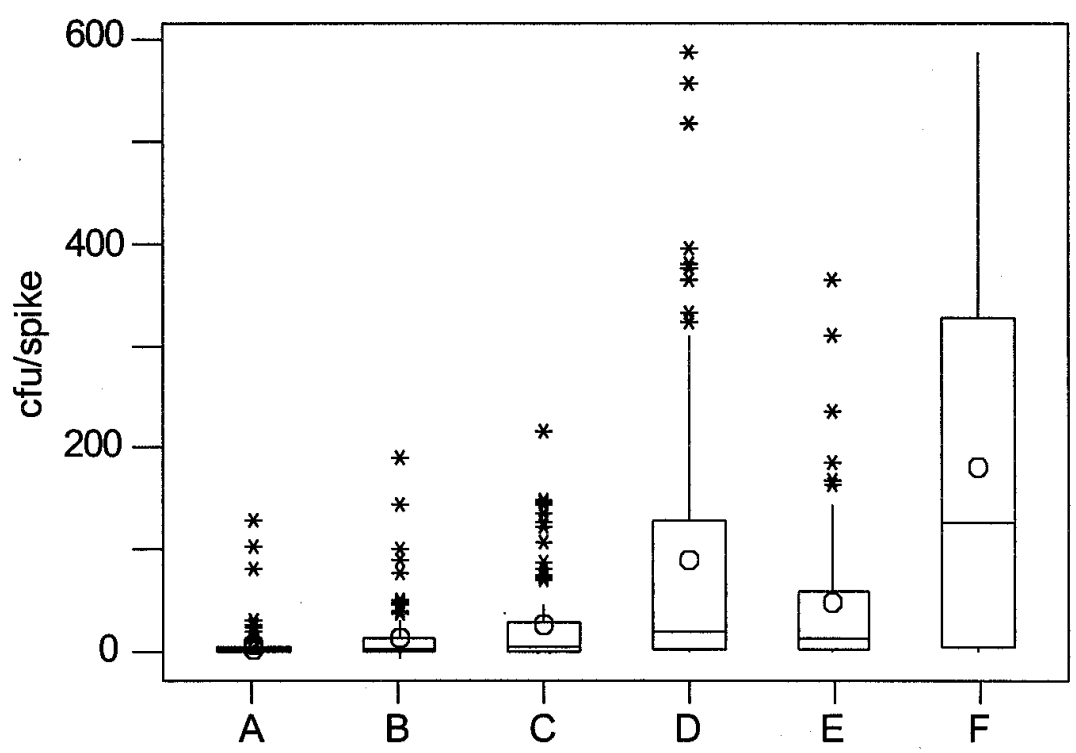

Fig. 2. Boxplots of daily inoculum levels of Gibberella zeae (CFU) on wheat spikes illustrating the two central quartiles (box), median (horizontal line), mean (circle), weak outliers (vertical line), and probable outliers (asterisks). Data categories plotted are samples from: remote locations in nonepidemic $(<10 \%$ disease severity) areas, A; remote locations in epidemic areas, B; field locations in nonepidemic areas, C; and field locations in an epidemic area, D. Field samples, D, were subdivided further into those from a field without corn residue, $\mathrm{E}$, and from a field with corn residue, $\mathrm{F}$. 
an experimental artifact. $F$. poae was found in Manitoba in a similar amount, 1,700 CFU per spike, late in the sampling period. We suspect the humid environment within the glassine bag allowed for sporulation and proliferation of fungi once introduced by insect or other happenstance.

Other Fusarium species were recovered from wheat spikes. F. culmorum has been associated with wheat head blight, while other species were less damaging (15). Although these species may contribute to disease severity and toxic contaminants of grain, management efforts should remain focused on $G$. zeae because of its abundance throughout North American wheat- and corn-growing regions. G. zeae is likely to remain a significant component of the aerial mycoflora of North America as long as conservation tillage is widely practiced and hosts are extensively cultivated.

\section{ACKNOWLEDGMENTS}

Research supported in part by the USDA-NRI Competitive Grants Program (Agreement 9501094) and the Minnesota Wheat Growers Research and Promotion Council. The authors express their appreciation to James Jordahl, Ronald Kaethler, David Kalb, Stan Kawamoto, Uwe Kromer, Bacilio Salas, and Christine Stockwell for their technical assistance.
LITERATURE CITED

1. Anderson, A. L. 1948. The development of Gibberella zeae headblight of wheat. Phytopathology 38:595-611.

2. Bai, G., and Shaner, G. 1994. Scab of wheat: Prospects for control. Plant Dis. 78:760-766.

3. Bennett, F. T. 1931. Gibberella saubinetii (Mont) Sacc. On British cereals. II. Physiological and pathological studies. Ann. Appl. Biol. 18:158-177.

4. Dhingra, O. D., and Sinclair, J. B. 1985. Basic Plant Pathology Methods. CRC Press, Boca Raton, FL.

5. Fernando, W. G. D., Paulitz, T. C., Seaman, W. L., Dutilleul, P., and Miller, J. D. 1997. Head blight gradients caused by Gibberella zeae from area sources of inoculum in wheat field plots. Phytopathology 87:414-421.

6. Khonga, E. B., and Sutton, J. C. 1988. Inoculum production and survival of Gibberella zeae in maize and wheat residues. Can. J. Plant Pathol. 10:232-239.

7. McMullen, M., Jones, R., and Gallenberg, D. 1997. Scab of wheat and barley: A re-emerging disease of devastating impact. Plant Dis. 81:1340-1348.

8. Miller, J. D. 1994. Epidemiology of Fusarium ear rot diseases of cereals. Pages 19-36 in: Mycotoxins in Grain: Compounds Other Than Aflatoxins. J. D. Miller and H. L. Trenholm, eds. American Association of Cereal Chemists, St. Paul, MN.

9. Moschini, R. C., and Fortugno, C. 1996. Predicting wheat head blight incidence using models based on meteorological factors in Pergamino, Argentina. Eur. J. Plant Pathol.
102:211-218.

10. Parry, D. W., Jenkinson, P., and McLeod, L. 1995. Fusarium ear blight (scab) in small grain cereals - a review. Plant Pathol. 44:207238.

11. Paulitz, T. C. 1996. Diurnal release of ascospores by Gibberella zeae in inoculated wheat plots. Plant Dis. 80:674-678.

12. Pritsch, C., Bushnell, W. R., Somers, D. A. Muehlbauer, G., and Vance, C. P. 1998. Fusarium graminearum infection on wheat spikes: Early events. Phytopathology 88:S73.

13. Schroeder, H. W., and Christiansen, J. J. 1963. Factors affecting resistance of wheat to scab caused by Gibberella zeae. Phytopathology 53:831-838.

14. Stack, R. W. 1989. A comparison of the inoculum potential of ascospores and conidia of Gibberella zeae. Can. J. Plant Pathol. 11:137142.

15. Stack, R. W., and McMullen, M. P. 1985. Head blighting potential of Fusarium species associated with spring wheat heads. Can. J. Plant Pathol. 7:79-82

16. Strange, R. N., and Smith, H. 1971. A fungal growth stimulant in anthers which predisposes wheat to attack by Fusarium graminearum. Physiol. Plant Pathol. 1:141150.

17. Sutton, J. C. 1982. Epidemiology of wheat head blight and maize ear rot caused by Fusarium graminearum. Can. J. Plant Pathol. 4:195-209.

18. Wilcoxson, R. D., Busch, R. H., and Ozmon, E. A. 1992. Fusarium head blight resistance in spring wheat cultivars. Plant Dis. 76:658-661. 